\title{
Evaluation of resazurin-based assay for rapid detection of polymyxin-resistant gram-negative bacteria
}

\author{
Huaiyu Jia ${ }^{1,2}$, Renchi Fang ${ }^{1}$, Jie Lin', Xuebin Tian², Yajie Zhao ${ }^{2}$, Lijiang Chen ${ }^{1}$, Jianming Cao ${ }^{2^{*}}$ and Tieli Zhou ${ }^{1 *}$ (D)
}

\begin{abstract}
Background: Colistin resistance is considered a serious problem due to a lack of alternative antibiotics. The Rapid ResaPolymyxin Acinetobacter/Pseudomonas NP test is a resazurin reduction-based technique that relies on the visual detection of bacterial growth in the presence of a defined concentration of colistin. The aim of this study was to evaluate the performance of the Rapid ResaPolymyxin Acinetobacter/Pseudomonas NP test in the detection of colistin susceptibility in common clinical Gram-negative bacteria.
\end{abstract}

Results: A total of 253 clinical isolates from a teaching hospital, including Acinetobacter baumanii $(n=58,8$ colistinresistant), Pseudomonas aeruginosa ( $n=61,11$ colistin-resistant), Klebsiella pneumoniae ( $n=70,20$ colistin-resistant) and Escherichia coli ( $n=64,14$ colistin-resistant) were tested in this study. The sensitivity and specificity of the Rapid ResaPolymyxin Acinetobacter/Pseudomonas NP test compared to Broth microdilution method was 100 and 99\%, respectively.

Conclusions: Our results suggest that Rapid ResaPolymyxin Acinetobacter/Pseudomonas NP test could be used as an accurate detection method for colistin resistance.

Keywords: Rapid ResaPolymyxin Acinetobacter/Pseudomonas NP test, Colistin-resistant, Gram-negative bacteria, Rapid diagnosis

\section{Background}

Polymyxin E, also known as colistin is a multicomponent polypeptide antibiotic, which belongs to the group of polymyxin [1]. Polymyxin E was discovered in the 1940s; yet, later on, it was abandoned in clinical practice due to its increased nephrotoxicity. However, due to the increase of multidrug resistance (MDR) in Gram-negative bacteria, especially in the ESKAPE pathogens (Enterococcus faecium, Staphylococcus aureus, Klebsiella pneumoniae, Acinetobacter baumannii, Pseudomonas aeruginosa, and Enterobacter species), colistin has been applied in clinical practice for the last few years as the last resort treatment option [2,3]. Currently, colistin resistance is considered a serious problem, due to a lack of alternative antibiotics $[4,5]$. As for now, rapid identification of colistin

\footnotetext{
* Correspondence: wzcjming@163.com; wyztli@163.com

${ }^{2}$ School of Laboratory Medicine and Life Sciences, Wenzhou Medical University, Wenzhou, Zhejiang Province, China

'Department of Clinical Laboratory, the First Affiliated Hospital of Wenzhou Medical University, Wenzhou, Zhejiang province, China
}

resistance is considered essential for the effective control of MDR Gram-negative bacteria infection.

Broth microdilution (BMD) is the only reference method that has been recommended by the European Committee on Antimicrobial Susceptibility Testing (EUCAST) and Clinical and Laboratory Standards Institute (CLSI) for the detection of minimum inhibitory concentrations (MICs) of colistin [6,7]. Nevertheless, colistin antimicrobial susceptibility testing is very challenging to perform $[8,9]$. For example, the operational steps of BMD are complex and time-consuming, making it unsuitable for clinical use [10]. Clinical microbiology laboratories are especially affected by the lack of an accurate, fast and easy-to-conduct method to test the colistin susceptibility [11-13]. Therefore, it is of great significance for clinical anti-infective treatment to develop and promote new, convenient, economical, rapid and accurate colistin sensitivity detection method.

In 2016, Nordmann et al developed the Rapid Polymyxins NP test for Enterobacteriaceae spp [14]. The

(c) The Author(s). 2020 Open Access This article is distributed under the terms of the Creative Commons Attribution 4.0 International License (http://creativecommons.org/licenses/by/4.0/), which permits unrestricted use, distribution, and 
method can be used to detect bacteria that can grow, metabolize glucose, and produce acid in the presence of polymyxin such as polymyxin $\mathrm{B}$ or colistin through color changes of $\mathrm{PH}$ indicators. However, one of the significant limitations when using this approach is that it cannot be applied for non-fermentative bacteria such as $A$. baumannii and $P$. aeruginosa. More recently, Lescat et al have developed a rapid resazurin-mucoid susceptibility test method called Rapid ResaPolymyxin Acinetobacter/Pseudomonas NP test, which can quickly detect the sensitivity of colistin for both Enterobacteriaceae spp and non-fermentative bacteria within $4 \mathrm{~h}$ [15]. The method is mainly based on detection of the strain viability by observing the color change of resazurin (an active colorant) from blue to purple or pink in the presence of colistin $(3.75 \mathrm{mg} / \mathrm{L})$.

In this study, we analyzed the performance of the Rapid ResaPolymyxin Acinetobacter/Pseudomonas NP test in the detection of colistin susceptibility in 253 nonduplicate clinical Gram-negative isolates aiming to provide a basis for the popularization and application of a new method for rapid screening of colistin-resistant common clinical Gram-negative bacteria.

\section{Results}

The colistin MICs of the 253 Gram-negative isolates ranged from $\leq 0.06$ to $\geq 32 \mathrm{mg} / \mathrm{L}$. BMD results were used as a standard, and 53 colistin-resistant strains and 198 colistin susceptible strains were correctly detected by the Rapid ResaPolymyxin Acinetobacter/Pseudomonas NP test. Very major errors (VME) and major errors (ME) corresponded to false-susceptible and false-resistant results, respectively [16]. There were only two ME in $A$. baumannii; details are shown in Tables 1 and 2. The specificity of $A$. baumannii was $96 \%$; while the sensitivity and specificity of the Rapid ResaPolymyxin Acinetobacter/Pseudomonas NP test to P. aeruginosa, K. pneumoniae and E. coli were $100 \%$ (Table 3).

\section{Discussion}

In this study, we described the diagnostic performance of the Rapid ResaPolymyxin Acinetobacter/Pseudomonas NP test, a phenotypic method for differentiation between colistin-resistant strains and colistin-susceptible strains. Compared with the reference BMD, the Rapid ResaPolymyxin Acinetobacter/Pseudomonas NP test showed accuracy in detecting the resistance to colistin. Besides, the method was fast, easy to perform, and the obtained data were easy to interpret. Rapid Polymyxin NP test makes up for the limitations of applicability in non-fermenters [14]. In our study, we examined it efficiency in detecting non-fermentative bacteria, but also fermentative bacteria, such as E. coli strains and K. pneumoniae strains. The results showed that the sensitivity and specificity of the Rapid ResaPolymyxin Acinetobacter/Pseudomonas $\mathrm{NP}$ test to Enterobacteriaceae were 100\%, which was consistent with a previous study [15]. In the present study, there were only two ME in colistin-susceptible $A$. baumannii strains. The categorical agreement for all tested isolates was $\mathbf{9 9 . 2 \%}$ for the Rapid ResaPolymyxin Acinetobacter/Pseudomonas NP test. In addition, the sensitivity and specificity were respectively 100 and $99 \%$, which further suggested that this method is suitable for detecting fermentative bacteria.

So far, a number of studies have examined the mechanism of colistin resistance $[17,18]$. This study revealed that chromosome mutations of two-component regulatory systems (TCSs) and $m c r-1$, which were located in plasmid, were the main causes of colistin resistance in 53 strains. In addition, we were able to detect drug resistance without a difference. Therefore, compared with the Rapid Polymyxin NP test, the Rapid ResaPolymyxin Acinetobacter/Pseudomonas NP test is suitable to be used in more scenes.

MicroScan Colistin Well is a newly developed kit for detection of colistin resistance in Gram-negative bacteria [19]. The fundamental principle of the Rapid ResaPolymyxin Acinetobacter/Pseudomonas NP test is similar to MicroScan Colistin Well. Both methods can be used to detect living bacteria in the medium with $4 \mathrm{mg} / \mathrm{L}$ or $3.75 \mathrm{mg} / \mathrm{L}$ of colistin (close to the breakpoint of colistin resistance). Similarly, the MICs cannot be determined utilizing the Rapid ResaPolymyxin Acinetobacter/Pseudomonas NP test and the MicroScan Colistin Well. Only colistin resistance results or sensitive test results can be obtained by them. However, there are two major differences between the two methods. First, Rapid ResaPolymyxin Acinetobacter/Pseudomonas NP test is significantly faster compared to MicroScan Colistin Well. For example, the detection of $P$. aeruginosa by Rapid ResaPolymyxin Acinetobacter/Pseudomonas NP test takes maximum $5 \mathrm{~h}$ to analyze the results, while MicroScan Colistin Well requires 16 to $18 \mathrm{~h}$. Secondly, in the presence of resazurin reagent PrestoBlue ${ }^{\circ}$, the growth of living bacteria of the Rapid ResaPolymyxin Acinetobacter/Pseudomonas NP test can be more clearly observed compared to MicroScan Colistin Well.

The principle of Rapid ResaPolymyxin Acinetobacter/ Pseudomonas NP test is based on the visual detection of the reduction of the resazurin reagent, a viability colorant that is observed by color change (blue to purple or pink). Interestingly, in the current study, no significant color changes were observed in colistin-resistant $P$. aeruginosa after the addition of the resazurin reagent for $1 \mathrm{~h}$. After prolonging the observation time for another $1 \mathrm{~h}$, the color changed from blue to purple. In other words, the results were not obtained until $2 \mathrm{~h}$ later in the study, while very obvious color changes were observed $15 \mathrm{~min}$ 
Table 1 Colistin MICs obtained by broth microdilution and results of the Rapid ResaPolymyxin Acinetobacter/Pseudomonas NP test

\begin{tabular}{|c|c|c|c|c|c|}
\hline \multirow[t]{2}{*}{ Isolate } & \multirow[t]{2}{*}{ Species } & \multirow{2}{*}{$\begin{array}{l}\text { Resistant } \\
\text { Phenotype }\end{array}$} & \multirow{2}{*}{$\begin{array}{l}\mathrm{MIC} \\
(\mathrm{mg} / \mathrm{L})\end{array}$} & \multicolumn{2}{|c|}{ Rapid ResaPolymyxin Acinetobacter/Pseudomonas NP Test } \\
\hline & & & & Result & $\begin{array}{l}\text { Discrepancies with BMD } \\
\text { MIC colistin result }\end{array}$ \\
\hline BM1539 & A. baumannii & $\mathrm{R}$ & 8 & Positive & No \\
\hline BM1579 & A. baumannii & $\mathrm{R}$ & 4 & Positive & No \\
\hline BM1595 & A. baumannii & $\mathrm{R}$ & 4 & Positive & No \\
\hline BM2349 & A. baumannii & $\mathrm{R}$ & 4 & Positive & No \\
\hline BM2370 & A. baumannii & $\mathrm{R}$ & 16 & Positive & No \\
\hline BM2412 & A. baumannii & $\mathrm{R}$ & 4 & Positive & No \\
\hline BM2431 & A. baumannii & $\mathrm{R}$ & 8 & Positive & No \\
\hline BM2622 & A. baumannii & $\mathrm{R}$ & 8 & Positive & No \\
\hline TL1671 & P. aeruginosa & $\mathrm{R}$ & 4 & Positive & No \\
\hline TL1722 & P. aeruginosa & $\mathrm{R}$ & 4 & Positive & No \\
\hline TL1736 & P. aeruginosa & $R$ & $\geq 32$ & Positive & No \\
\hline TL1744 & P. aeruginosa & $\mathrm{R}$ & 4 & Positive & No \\
\hline TL2204 & P. aeruginosa & $R$ & 4 & Positive & No \\
\hline TL2294 & P. aeruginosa & $\mathrm{R}$ & 4 & Positive & No \\
\hline TL2314 & P. aeruginosa & $\mathrm{R}$ & $\geq 32$ & Positive & No \\
\hline TL2917 & P. aeruginosa & $\mathrm{R}$ & 4 & Positive & No \\
\hline TL2967 & P. aeruginosa & $\mathrm{R}$ & 4 & Positive & No \\
\hline TL3008 & P. aeruginosa & $\mathrm{R}$ & 16 & Positive & No \\
\hline TL3086 & P. aeruginosa & $\mathrm{R}$ & $\geq 32$ & Positive & No \\
\hline FK20 & K. pneumoniae & $\mathrm{R}$ & $\geq 32$ & Positive & No \\
\hline FK26 & K. pneumoniae & $\mathrm{R}$ & $\geq 32$ & Positive & No \\
\hline FK150 & K. pneumoniae & $\mathrm{R}$ & $\geq 32$ & Positive & No \\
\hline FK169 & K. pneumoniae & $\mathrm{R}$ & $\geq 32$ & Positive & No \\
\hline FK171 & K. pneumoniae & $\mathrm{R}$ & $\geq 32$ & Positive & No \\
\hline FK591 & K. pneumoniae & $\mathrm{R}$ & $\geq 32$ & Positive & No \\
\hline FK610 & K. pneumoniae & $\mathrm{R}$ & $\geq 32$ & Positive & No \\
\hline FK1342 & K. pneumoniae & $\mathrm{R}$ & $\geq 32$ & Positive & No \\
\hline FK1913 & K. pneumoniae & R & $\geq 32$ & Positive & No \\
\hline FK1986 & K. pneumoniae & $\mathrm{R}$ & 8 & Positive & No \\
\hline FK2066 & K. pneumoniae & $\mathrm{R}$ & $\geq 32$ & Positive & No \\
\hline FK2166 & K. pneumoniae & $\mathrm{R}$ & $\geq 32$ & Positive & No \\
\hline FK2778 & K. pneumoniae & $R$ & $\geq 32$ & Positive & No \\
\hline FK2911 & K. pneumoniae & $\mathrm{R}$ & $\geq 32$ & Positive & No \\
\hline FK3789 & K. pneumoniae & $R$ & $\geq 32$ & Positive & No \\
\hline FK3810 & K. pneumoniae & $\mathrm{R}$ & $\geq 32$ & Positive & No \\
\hline FK3994 & K. pneumoniae & $\mathrm{R}$ & $\geq 32$ & Positive & No \\
\hline FK6556 & K. pneumoniae & $\mathrm{R}$ & 32 & Positive & No \\
\hline FK6663 & K. pneumoniae & $\mathrm{R}$ & 32 & Positive & No \\
\hline FK6696 & K. pneumoniae & $\mathrm{R}$ & 16 & Positive & No \\
\hline DC90 & E. coli & R & 8 & Positive & No \\
\hline DC2562 & E. coli & $\mathrm{R}$ & 8 & Positive & No \\
\hline DC3411 & E. coli & R & 4 & Positive & No \\
\hline DC3539 & E. coli & $\mathrm{R}$ & 16 & Positive & No \\
\hline
\end{tabular}


Table 1 Colistin MICs obtained by broth microdilution and results of the Rapid ResaPolymyxin Acinetobacter/Pseudomonas NP test (Continued)

\begin{tabular}{|c|c|c|c|c|c|}
\hline \multirow[t]{2}{*}{ Isolate } & \multirow[t]{2}{*}{ Species } & \multirow{2}{*}{$\begin{array}{l}\text { Resistant } \\
\text { Phenotype }\end{array}$} & \multirow{2}{*}{$\begin{array}{l}\mathrm{MIC} \\
(\mathrm{mg} / \mathrm{L})\end{array}$} & \multicolumn{2}{|c|}{ Rapid ResaPolymyxin Acinetobacter/Pseudomonas NP Tes } \\
\hline & & & & Result & $\begin{array}{l}\text { Discrepancies with BMD } \\
\text { MIC colistin result }\end{array}$ \\
\hline DC3599 & E. coli & $\mathrm{R}$ & 8 & Positive & No \\
\hline DC3658 & E. coli & $\mathrm{R}$ & 8 & Positive & No \\
\hline DC3737 & E. coli & $\mathrm{R}$ & 8 & Positive & No \\
\hline DC3802 & E. coli & $\mathrm{R}$ & 4 & Positive & No \\
\hline DC3806 & E. coli & $\mathrm{R}$ & 8 & Positive & No \\
\hline DC3846 & E. coli & $\mathrm{R}$ & 16 & Positive & No \\
\hline DC4887 & E. coli & $\mathrm{R}$ & 8 & Positive & No \\
\hline DC5262 & E. coli & $\mathrm{R}$ & 8 & Positive & No \\
\hline DC5286 & E. coli & $\mathrm{R}$ & 8 & Positive & No \\
\hline DC7333 & E. coli & $\mathrm{R}$ & 4 & Positive & No \\
\hline BM1505 & A. baumannii & $\mathrm{S}$ & 0.125 & Negative & No \\
\hline BM1506 & A. baumannii & S & 0.5 & Negative & No \\
\hline BM1507 & A. baumannii & $\mathrm{S}$ & 0.06 & Negative & No \\
\hline BM1508 & A. baumannii & S & 0.125 & Negative & No \\
\hline BM1509 & A. baumannii & S & 0.125 & Negative & No \\
\hline BM1510 & A. baumannii & S & 0.125 & Negative & No \\
\hline BM1511 & A. baumannii & $S$ & 0.125 & Negative & No \\
\hline BM1512 & A. baumannii & S & 0.25 & Negative & No \\
\hline BM1513 & A. baumannii & S & 0.125 & Negative & No \\
\hline BM1514 & A. baumannii & S & 0.125 & Negative & No \\
\hline BM4151 & A. baumannii & S & 0.25 & Negative & No \\
\hline BM4152 & A. baumannii & S & 0.06 & Negative & No \\
\hline BM4153 & A. baumannii & S & 0.03 & Negative & No \\
\hline BM4154 & A. baumannii & S & 0.125 & Negative & No \\
\hline BM4155 & A. baumannii & $\mathrm{S}$ & 0.125 & Negative & No \\
\hline BM4156 & A. baumannii & S & 0.125 & Negative & No \\
\hline BM4158 & A. baumannii & $\mathrm{S}$ & 0.125 & Negative & No \\
\hline BM4159 & A. baumannii & S & 0.125 & Negative & No \\
\hline BM4160 & A. baumannii & S & 0.5 & Negative & No \\
\hline BM4161 & A. baumannii & S & 0.125 & Negative & No \\
\hline BM4162 & A. baumannii & S & 0.06 & Negative & No \\
\hline BM4163 & A. baumannii & S & 0.06 & Negative & No \\
\hline BM4164 & A. baumannii & S & 0.06 & Negative & No \\
\hline BM4165 & A. baumannii & $\mathrm{S}$ & 0.06 & Negative & No \\
\hline BM4166 & A. baumannii & S & 0.125 & Negative & No \\
\hline BM4167 & A. baumannii & S & 0.125 & Negative & No \\
\hline BM4168 & A. baumannii & S & 0.25 & Negative & No \\
\hline BM4169 & A. baumannii & S & 0.5 & Negative & No \\
\hline BM4170 & A. baumannii & S & 0.125 & Negative & No \\
\hline BM4171 & A. baumannii & S & 0.06 & Negative & No \\
\hline BM4172 & A. baumannii & $\mathrm{S}$ & $\leq 0.06$ & Negative & No \\
\hline BM4173 & A. baumannii & S & 0.06 & Negative & No \\
\hline BM4174 & A. baumannii & $\mathrm{S}$ & 0.06 & Negative & No \\
\hline
\end{tabular}


Table 1 Colistin MICs obtained by broth microdilution and results of the Rapid ResaPolymyxin Acinetobacter/Pseudomonas NP test (Continued)

\begin{tabular}{|c|c|c|c|c|c|}
\hline \multirow[t]{2}{*}{ Isolate } & \multirow[t]{2}{*}{ Species } & \multirow{2}{*}{$\begin{array}{l}\text { Resistant } \\
\text { Phenotype }\end{array}$} & \multirow{2}{*}{$\begin{array}{l}\mathrm{MIC} \\
(\mathrm{mg} / \mathrm{L})\end{array}$} & \multicolumn{2}{|c|}{ Rapid ResaPolymyxin Acinetobacter/Pseudomonas NP Test } \\
\hline & & & & Result & $\begin{array}{l}\text { Discrepancies with BMD } \\
\text { MIC colistin result }\end{array}$ \\
\hline BM4175 & A. baumannii & S & 2 & Negative & No \\
\hline BM4176 & A. baumannii & S & 0.06 & Negative & No \\
\hline BM4177 & A. baumannii & S & 0.06 & Negative & No \\
\hline BM4178 & A. baumannii & $\mathrm{S}$ & 0.06 & Negative & No \\
\hline BM4179 & A. baumannii & S & 0.25 & Negative & No \\
\hline BM4180 & A. baumannii & $\mathrm{S}$ & 0.06 & Negative & No \\
\hline BM4181 & A. baumannii & S & 0.125 & Negative & No \\
\hline BM4182 & A. baumannii & S & 0.25 & Negative & No \\
\hline BM4183 & A. baumannii & S & 1 & Negative & No \\
\hline BM4184 & A. baumannii & S & 1 & Positive & Yes, ME \\
\hline BM4185 & A. baumannii & $\mathrm{S}$ & 1 & Negative & No \\
\hline BM4186 & A. baumannii & S & 1 & Negative & No \\
\hline BM4187 & A. baumannii & $\mathrm{S}$ & 0.125 & Negative & No \\
\hline BM4188 & A. baumannii & S & 0.5 & Positive & Yes, ME \\
\hline BM4189 & A. baumannii & S & 0.5 & Negative & No \\
\hline BM4190 & A. baumannii & S & 0.125 & Negative & No \\
\hline BM4191 & A. baumannii & S & 0.5 & Negative & No \\
\hline TL2916 & P. aeruginosa & S & 0.125 & Negative & No \\
\hline TL2915 & P. aeruginosa & S & $\leq 0.06$ & Negative & No \\
\hline TL2914 & P. aeruginosa & S & 0.125 & Negative & No \\
\hline TL2913 & P. aeruginosa & S & 0.125 & Negative & No \\
\hline TL2911 & P. aeruginosa & S & 0.125 & Negative & No \\
\hline TL2910 & P. aeruginosa & S & 0.25 & Negative & No \\
\hline TL2908 & P. aeruginosa & S & 0.125 & Negative & No \\
\hline TL2907 & P. aeruginosa & $\mathrm{S}$ & 0.5 & Negative & No \\
\hline TL2906 & P. aeruginosa & S & 0.125 & Negative & No \\
\hline TL2905 & P. aeruginosa & $\mathrm{S}$ & 0.125 & Negative & No \\
\hline TL2904 & P. aeruginosa & S & 0.125 & Negative & No \\
\hline TL2901 & P. aeruginosa & S & 0.125 & Negative & No \\
\hline TL2899 & P. aeruginosa & S & 0.125 & Negative & No \\
\hline TL2898 & P. aeruginosa & S & 0.125 & Negative & No \\
\hline TL2897 & P. aeruginosa & S & 0.125 & Negative & No \\
\hline TL2895 & P. aeruginosa & S & 0.125 & Negative & No \\
\hline TL2893 & P. aeruginosa & $\mathrm{S}$ & $\leq 0.06$ & Negative & No \\
\hline TL2892 & P. aeruginosa & S & 0.125 & Negative & No \\
\hline TL2891 & P. aeruginosa & S & 0.06 & Negative & No \\
\hline TL2890 & P. aeruginosa & S & 0.25 & Negative & No \\
\hline TL2889 & P. aeruginosa & S & 0.5 & Negative & No \\
\hline TL2886 & P. aeruginosa & S & 0.5 & Negative & No \\
\hline TL2885 & P. aeruginosa & S & 0.25 & Negative & No \\
\hline TL2884 & P. aeruginosa & $\mathrm{S}$ & 0.25 & Negative & No \\
\hline TL2883 & P. aeruginosa & S & 0.5 & Negative & No \\
\hline TL2882 & P. aeruginosa & $\mathrm{S}$ & 0.25 & Negative & No \\
\hline
\end{tabular}


Table 1 Colistin MICs obtained by broth microdilution and results of the Rapid ResaPolymyxin Acinetobacter/Pseudomonas NP test (Continued)

\begin{tabular}{|c|c|c|c|c|c|}
\hline \multirow[t]{2}{*}{ Isolate } & \multirow[t]{2}{*}{ Species } & \multirow{2}{*}{$\begin{array}{l}\text { Resistant } \\
\text { Phenotype }\end{array}$} & \multirow{2}{*}{$\begin{array}{l}\mathrm{MIC} \\
(\mathrm{mg} / \mathrm{L})\end{array}$} & \multicolumn{2}{|c|}{ Rapid ResaPolymyxin Acinetobacter/Pseudomonas NP Test } \\
\hline & & & & Result & $\begin{array}{l}\text { Discrepancies with BMD } \\
\text { MIC colistin result }\end{array}$ \\
\hline TL2881 & P. aeruginosa & S & 0.25 & Negative & No \\
\hline TL2879 & P. aeruginosa & S & 0.25 & Negative & No \\
\hline TL2878 & P. aeruginosa & S & 0.25 & Negative & No \\
\hline TL2877 & P. aeruginosa & $\mathrm{S}$ & 0.25 & Negative & No \\
\hline TL2875 & P. aeruginosa & S & 0.25 & Negative & No \\
\hline TL2874 & P. aeruginosa & $\mathrm{S}$ & 0.25 & Negative & No \\
\hline TL2873 & P. aeruginosa & S & 1 & Negative & No \\
\hline TL2872 & P. aeruginosa & S & 0.125 & Negative & No \\
\hline TL2871 & P. aeruginosa & S & 0.25 & Negative & No \\
\hline TL2870 & P. aeruginosa & S & 2 & Negative & No \\
\hline TL2869 & P. aeruginosa & $\mathrm{S}$ & 0.125 & Negative & No \\
\hline TL2868 & P. aeruginosa & S & 0.125 & Negative & No \\
\hline TL2867 & P. aeruginosa & S & 0.125 & Negative & No \\
\hline TL2866 & P. aeruginosa & S & 0.125 & Negative & No \\
\hline TL2865 & P. aeruginosa & S & 0.125 & Negative & No \\
\hline TL2864 & P. aeruginosa & S & 0.25 & Negative & No \\
\hline TL2863 & P. aeruginosa & S & $\leq 0.06$ & Negative & No \\
\hline TL2862 & P. aeruginosa & S & 0.125 & Negative & No \\
\hline TL2861 & $P$. aeruginosa & S & 0.25 & Negative & No \\
\hline TL2858 & P. aeruginosa & S & 0.25 & Negative & No \\
\hline TL2857 & P. aeruginosa & S & 0.25 & Negative & No \\
\hline TL2856 & P. aeruginosa & S & 0.125 & Negative & No \\
\hline TL2855 & P. aeruginosa & S & 0.25 & Negative & No \\
\hline TL2854 & P. aeruginosa & S & 0.125 & Negative & No \\
\hline FK3640 & K. pneumoniae & $\mathrm{S}$ & $\leq 0.06$ & Negative & No \\
\hline FK3642 & K. pneumoniae & S & $\leq 0.06$ & Negative & No \\
\hline FK3646 & K. pneumoniae & $\mathrm{S}$ & $\leq 0.06$ & Negative & No \\
\hline FK3660 & K. pneumoniae & S & $\leq 0.06$ & Negative & No \\
\hline FK3671 & K. pneumoniae & S & 0.125 & Negative & No \\
\hline FK3686 & K. pneumoniae & S & 0.5 & Negative & No \\
\hline FK3695 & K. pneumoniae & S & $\leq 0.06$ & Negative & No \\
\hline FK3696 & K. pneumoniae & $S$ & $\leq 0.06$ & Negative & No \\
\hline FK3703 & K. pneumoniae & S & $\leq 0.06$ & Negative & No \\
\hline FK3712 & K. pneumoniae & $\mathrm{S}$ & $\leq 0.06$ & Negative & No \\
\hline FK3719 & K. pneumoniae & S & $\leq 0.06$ & Negative & No \\
\hline FK3721 & K. pneumoniae & S & $\leq 0.06$ & Negative & No \\
\hline FK3724 & K. pneumoniae & S & 1 & Negative & No \\
\hline FK3727 & K. pneumoniae & S & 0.5 & Negative & No \\
\hline FK3730 & K. pneumoniae & S & $\leq 0.06$ & Negative & No \\
\hline FK3732 & K. pneumoniae & S & 0.25 & Negative & No \\
\hline FK3738 & K. pneumoniae & $\mathrm{S}$ & $\leq 0.06$ & Negative & No \\
\hline FK3739 & K. pneumoniae & S & 0.125 & Negative & No \\
\hline FK3740 & K. pneumoniae & S & $\leq 0.06$ & Negative & No \\
\hline
\end{tabular}


Table 1 Colistin MICs obtained by broth microdilution and results of the Rapid ResaPolymyxin Acinetobacter/Pseudomonas NP test (Continued)

\begin{tabular}{|c|c|c|c|c|c|}
\hline \multirow[t]{2}{*}{ Isolate } & \multirow[t]{2}{*}{ Species } & \multirow{2}{*}{$\begin{array}{l}\text { Resistant } \\
\text { Phenotype }\end{array}$} & \multirow{2}{*}{$\begin{array}{l}\mathrm{MIC} \\
(\mathrm{mg} / \mathrm{L})\end{array}$} & \multicolumn{2}{|c|}{ Rapid ResaPolymyxin Acinetobacter/Pseudomonas NP Test } \\
\hline & & & & Result & $\begin{array}{l}\text { Discrepancies with BMD } \\
\text { MIC colistin result }\end{array}$ \\
\hline FK3741 & K. pneumoniae & S & $\leq 0.06$ & Negative & No \\
\hline FK3745 & K. pneumoniae & S & 0.5 & Negative & No \\
\hline FK3746 & K. pneumoniae & S & 1 & Negative & No \\
\hline FK3749 & K. pneumoniae & $\mathrm{S}$ & $\leq 0.06$ & Negative & No \\
\hline FK3758 & K. pneumoniae & S & $\leq 0.06$ & Negative & No \\
\hline FK3764 & K. pneumoniae & $\mathrm{S}$ & $\leq 0.06$ & Negative & No \\
\hline FK3767 & K. pneumoniae & S & $\leq 0.06$ & Negative & No \\
\hline FK3771 & K. pneumoniae & S & 0.5 & Negative & No \\
\hline FK3784 & K. pneumoniae & S & $\leq 0.06$ & Negative & No \\
\hline FK3800 & K. pneumoniae & S & 0.5 & Negative & No \\
\hline FK3803 & K. pneumoniae & $\mathrm{S}$ & 0.25 & Negative & No \\
\hline FK3813 & K. pneumoniae & S & $\leq 0.06$ & Negative & No \\
\hline FK3817 & K. pneumoniae & S & $\leq 0.06$ & Negative & No \\
\hline FK3824 & K. pneumoniae & S & 0.06 & Negative & No \\
\hline FK3830 & K. pneumoniae & S & $\leq 0.06$ & Negative & No \\
\hline FK3831 & K. pneumoniae & S & $\leq 0.06$ & Negative & No \\
\hline FK3838 & K. pneumoniae & S & 0.5 & Negative & No \\
\hline FK3844 & K. pneumoniae & S & 0.125 & Negative & No \\
\hline FK3853 & K. pneumoniae & S & 0.125 & Negative & No \\
\hline FK3878 & K. pneumoniae & S & 0.25 & Negative & No \\
\hline FK3882 & K. pneumoniae & S & $\leq 0.06$ & Negative & No \\
\hline FK3891 & K. pneumoniae & S & $\leq 0.06$ & Negative & No \\
\hline FK3927 & K. pneumoniae & S & 0.125 & Negative & No \\
\hline FK3938 & K. pneumoniae & S & 0.5 & Negative & No \\
\hline FK3943 & K. pneumoniae & S & 0.06 & Negative & No \\
\hline FK3946 & K. pneumoniae & S & 0.5 & Negative & No \\
\hline FK3989 & K. pneumoniae & $\mathrm{S}$ & $\leq 0.06$ & Negative & No \\
\hline FK3990 & K. pneumoniae & S & 1 & Negative & No \\
\hline FK3996 & K. pneumoniae & S & 0.125 & Negative & No \\
\hline FK3999 & K. pneumoniae & S & $\leq 0.06$ & Negative & No \\
\hline FK4002 & K. pneumoniae & S & $\leq 0.06$ & Negative & No \\
\hline DC8640 & E. coli & S & 0.25 & Negative & No \\
\hline DC8641 & E. coli & S & $\leq 0.06$ & Negative & No \\
\hline DC8642 & E. coli & $\mathrm{S}$ & 0.125 & Negative & No \\
\hline DC8643 & E. coli & S & 0.125 & Negative & No \\
\hline DC8644 & E. coli & S & 0.5 & Negative & No \\
\hline DC8645 & E. coli & S & $\leq 0.06$ & Negative & No \\
\hline DC8646 & E. coli & S & $\leq 0.06$ & Negative & No \\
\hline DC8647 & E. coli & S & $\leq 0.06$ & Negative & No \\
\hline DC8648 & E. coli & S & $\leq 0.06$ & Negative & No \\
\hline DC8649 & E. coli & $\mathrm{S}$ & 0.125 & Negative & No \\
\hline DC8650 & E. coli & S & 0.06 & Negative & No \\
\hline DC8651 & E. coli & $\mathrm{S}$ & 0.06 & Negative & No \\
\hline
\end{tabular}


Table 1 Colistin MICs obtained by broth microdilution and results of the Rapid ResaPolymyxin Acinetobacter/Pseudomonas NP test (Continued)

\begin{tabular}{|c|c|c|c|c|c|}
\hline \multirow[t]{2}{*}{ Isolate } & \multirow[t]{2}{*}{ Species } & \multirow{2}{*}{$\begin{array}{l}\text { Resistant } \\
\text { Phenotype }\end{array}$} & \multirow{2}{*}{$\begin{array}{l}\mathrm{MIC} \\
(\mathrm{mg} / \mathrm{L})\end{array}$} & \multicolumn{2}{|c|}{ Rapid ResaPolymyxin Acinetobacter/Pseudomonas NP Test } \\
\hline & & & & Result & $\begin{array}{l}\text { Discrepancies with BMD } \\
\text { MIC colistin result }\end{array}$ \\
\hline$\overline{D C 8652}$ & E. coli & $\mathrm{S}$ & 0.125 & Negative & No \\
\hline DC8653 & E. coli & $\mathrm{S}$ & 0.06 & Negative & No \\
\hline DC8654 & E. coli & $\mathrm{S}$ & 0.06 & Negative & No \\
\hline DC8655 & E. coli & $\mathrm{S}$ & 0.06 & Negative & No \\
\hline DC8656 & E. coli & $S$ & 0.125 & Negative & No \\
\hline DC8657 & E. coli & $\mathrm{S}$ & $\leq 0.06$ & Negative & No \\
\hline DC8658 & E. coli & $S$ & 0.06 & Negative & No \\
\hline DC8659 & E. coli & $S$ & $\leq 0.06$ & Negative & No \\
\hline DC8660 & E. coli & $S$ & $\leq 0.06$ & Negative & No \\
\hline DC8661 & E. coli & $S$ & $\leq 0.06$ & Negative & No \\
\hline DC8663 & E. coli & $S$ & 0.125 & Negative & No \\
\hline DC8664 & E. coli & $S$ & 2 & Negative & No \\
\hline DC8665 & E. coli & $S$ & $\leq 0.06$ & Negative & No \\
\hline DC8666 & E. coli & $\mathrm{S}$ & 0.06 & Negative & No \\
\hline DC8667 & E. coli & $S$ & $\leq 0.06$ & Negative & No \\
\hline DC8668 & E. coli & $S$ & $\leq 0.06$ & Negative & No \\
\hline DC8669 & E. coli & $S$ & $\leq 0.06$ & Negative & No \\
\hline DC8670 & E. coli & $\mathrm{S}$ & $\leq 0.06$ & Negative & No \\
\hline DC8671 & E. coli & $S$ & $\leq 0.06$ & Negative & No \\
\hline DC8672 & E. coli & $S$ & $\leq 0.06$ & Negative & No \\
\hline DC8673 & E. coli & $\mathrm{S}$ & $\leq 0.06$ & Negative & No \\
\hline DC8674 & E. coli & $S$ & 0.06 & Negative & No \\
\hline DC8675 & E. coli & $S$ & $\leq 0.06$ & Negative & No \\
\hline DC8676 & E. coli & $S$ & $\leq 0.06$ & Negative & No \\
\hline DC8677 & E. coli & $S$ & $\leq 0.06$ & Negative & No \\
\hline DC8678 & E. coli & $S$ & $\leq 0.06$ & Negative & No \\
\hline DC8679 & E. coli & $S$ & $\leq 0.06$ & Negative & No \\
\hline DC8680 & E. coli & $S$ & 0.25 & Negative & No \\
\hline DC8681 & E. coli & $S$ & 2 & Negative & No \\
\hline DC8682 & E. coli & $S$ & 0.125 & Negative & No \\
\hline DC8683 & E. coli & $S$ & $\leq 0.06$ & Negative & No \\
\hline DC8684 & E. coli & $S$ & $\leq 0.06$ & Negative & No \\
\hline DC8685 & E. coli & $S$ & $\leq 0.06$ & Negative & No \\
\hline DC8686 & E. coli & $S$ & 0.06 & Negative & No \\
\hline DC8687 & E. coli & $S$ & 0.06 & Negative & No \\
\hline DC8688 & E. coli & $S$ & 0.06 & Negative & No \\
\hline DC8690 & E. coli & $S$ & 0.06 & Negative & No \\
\hline DC8691 & E. coli & $\mathrm{S}$ & 0.125 & Negative & No \\
\hline
\end{tabular}

$M E$ major error, $S$ susceptible, $R$ resistant

after the addition of the resazurin reagent in the colistinresistant strains of $A$. baumanii, $K$. pneumoniae and $E$. coli, including $2 \mathrm{ME}$. This may be because the growth rate of $P$. aeruginosa is slower than that of Enterobacteriaceae, thus taking longer to decompose resazurin into fluorescent substance resorufin. It suggested that the observation time of the results of this experiment needed to be optimized according to the strain. 
Table 2 Colistin MICs for 253 Gram-negative isolates

\begin{tabular}{|c|c|c|c|c|c|c|c|c|c|c|c|}
\hline \multirow[t]{2}{*}{ Organism } & \multirow{2}{*}{$\begin{array}{l}\text { Number of } \\
\text { isolates }\end{array}$} & \multicolumn{10}{|c|}{ Colistin MIC (mg/L) } \\
\hline & & $\leq 0.06$ & 0.125 & 0.25 & 0.5 & 1 & 2 & 4 & 8 & 16 & $\geq 32$ \\
\hline Total & 253 & 86 & 56 & 27 & 19 & 8 & 4 & 14 & 13 & 5 & 21 \\
\hline A. baumannii & 58 & 15 & 19 & 5 & 6 & 4 & 1 & 4 & 3 & 1 & 0 \\
\hline P. aeruginosa & 61 & 4 & 23 & 17 & 4 & 1 & 1 & 7 & 0 & 1 & 3 \\
\hline K. pneumoniae & 70 & 30 & 6 & 3 & 8 & 3 & 0 & 0 & 1 & 1 & 18 \\
\hline E. coli & 64 & 37 & 8 & 2 & 1 & 0 & 2 & 3 & 9 & 2 & 0 \\
\hline
\end{tabular}

However, the Rapid ResaPolymyxin Acinetobacter/ Pseudomonas NP test still has some limitations. Firstly, the accurate MIC values could not be obtained. Since the Rapid ResaPolymyxin Acinetobacter/Pseudomonas NP test was not suitable for the study of high-level drug resistant strains, the method could only show whether the colistin resistant was present or not. Secondly, several $m c r$-harboring isolates with an MIC of $2 \mathrm{mg} / \mathrm{L}$ (or even less) to colistin or polymyxin $\mathrm{B}$ have been reported $[20,21]$, while our method could only be used to screen colistin resistant strains with $\mathrm{MIC} \geq 4 \mathrm{mg} / \mathrm{L}$. Thirdly, the reading time of $P$. aeruginosa results was different from that reported by the inventors, requiring an additional 1 $h$ of observation time.

\section{Conclusion}

The Rapid ResaPolymyxin Acinetobacter/Pseudomonas NP test has great stability and sensitivity in detection of colistin resistance in Gram-negative bacteria such as $A$. baumanii, $P$. aeruginosa, $K$. pneumoniae and $E$. coli strains. In addition, this method is fast and easy to perform. It can contribute in selecting more precise therapeutic choices, and optimizing antibiotic stewardship, and preventing the development of outbreaks with multidrug-resistant isolates. Nevertheless, the testing time of $P$. aeruginosa is longer than that reported by the inventor, so the observation time of this method needs to be further optimized.

\section{Methods}

\section{Bacterial strains}

A total of 253 nonduplicate clinical Gram-negative isolates including $A$. baumanii strains $(n=58)$, $P$. aeruginosa strains $(n=61), K$. pneumoniae strains $(n=70)$ and $E$. coli strains $(n=64)$ were obtained from a teaching hospital in Wenzhou, China. Species identification was performed using the Matrix-Assisted Laser Desorption Ionization Time-Of-Flight Mass Spectrometry (MALDITOF MS, Bruker Daltonics, US). A total of 53 colistinresistant strains were selected from our previous studies and were detected by BMD, including $8 \mathrm{~A}$. baumanii strains, $11 P$. aeruginosa strains, $20 \mathrm{~K}$. pneumoniae strains and $14 \mathrm{E}$. coli strains. In addition, 50 colistinsusceptible isolates of each four bacterial species mentioned above were randomly selected as the control group. E. coli ATCC 25922 and P. aeruginosa ATCC 27853 were used as control strains [6].

\section{Antimicrobial susceptibility test}

BMD was performed in triplicate. According to the EUCAST/CLSI joined guidelines $[6,7]$, the clinical breakpoints for colistin provided for $P$. aeruginosa and A. baumanii were $\leq 2 \mathrm{mg} / \mathrm{L}$ (susceptible breakpoint) and $\geq 4 \mathrm{mg} / \mathrm{L}$ (resistant breakpoint) and Enterobacteriaceae are $\leq 2 \mathrm{mg} / \mathrm{L}$ (susceptible breakpoint) and $>2 \mathrm{mg} / \mathrm{L}$ (resistant breakpoint).

Table 3 Rapid ResaPolymyxin Acinetobacter/Pseudomonas NP test results among Gram-negative isolates

\begin{tabular}{|c|c|c|c|c|c|c|}
\hline Organism & $\begin{array}{l}\text { Susceptibility to } \\
\text { polymyxins }\end{array}$ & Resistance mechanism & Isolates & $\begin{array}{l}\text { Rapid ResaPolymyxin Acinetobacter/ } \\
\text { Pseudomonas NP test }\end{array}$ & Sensitivity & Specificity \\
\hline \multirow[t]{2}{*}{ A. baumannii } & Resistant & Mediated by chromosome $e^{a}$ & $8(3.16 \%)$ & 8 positive result & $100 \%$ & $96 \%$ \\
\hline & Susceptible & & $50(19.76 \%)$ & $\begin{array}{l}48 \text { negative results and } 2 \\
\text { positive result }\end{array}$ & & \\
\hline \multirow[t]{2}{*}{ P. aeruginosa } & Resistant & Mediated by chromosome & $11(4.35 \%)$ & 11 positive result & $100 \%$ & $100 \%$ \\
\hline & Susceptible & & $50(19.76 \%)$ & 50 negative results & & \\
\hline \multirow[t]{2}{*}{ K. pneumoniae } & Resistant & Mediated by chromosome ${ }^{a}$ & $20(7.91 \%)$ & 20 positive result & $100 \%$ & $100 \%$ \\
\hline & Susceptible & & $50(19.76 \%)$ & 50 negative results & & \\
\hline \multirow[t]{2}{*}{ E. coli } & Resistant & Mediated by plasmid & $14(5.54 \%)$ & 2 positive result & $100 \%$ & $100 \%$ \\
\hline & Susceptible & & $50(19.76 \%)$ & 50 negative results & & \\
\hline
\end{tabular}




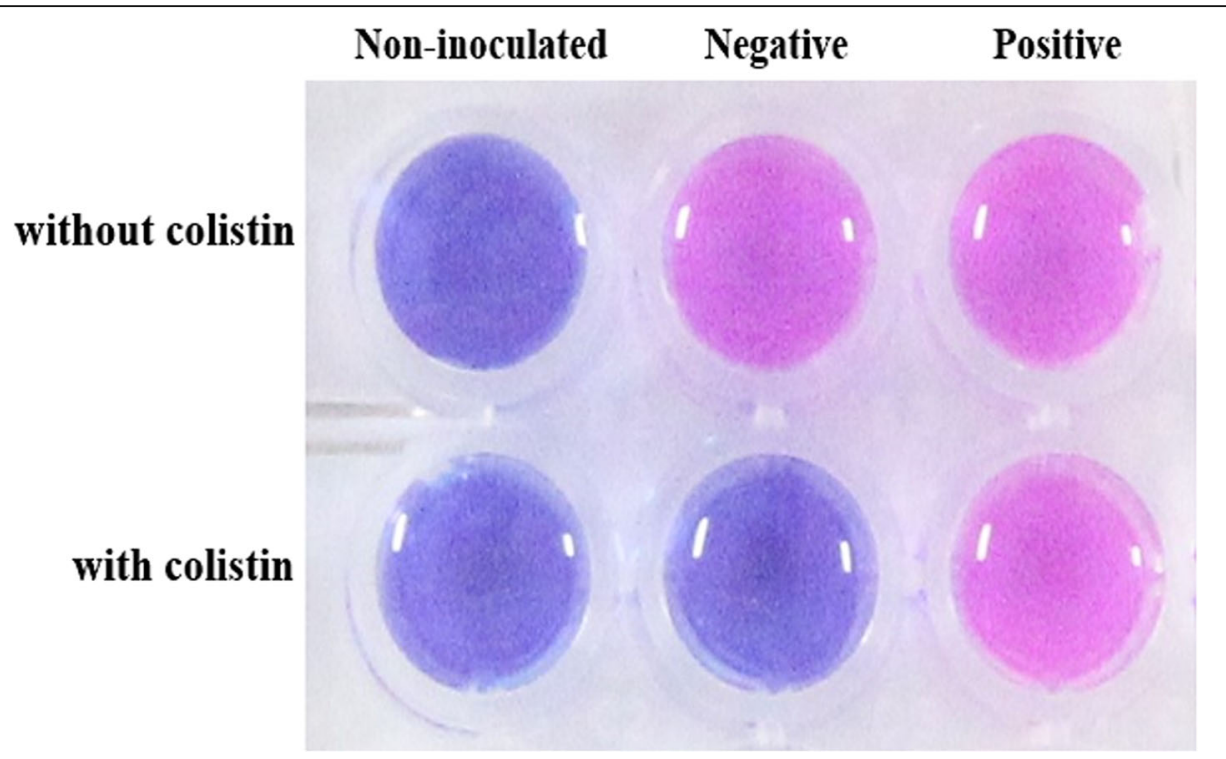

Fig. 1 Representative results of the Rapid ResaPolymyxin Acinetobacter/Pseudomonas NP test. Non-inoculated well is shown as the control of the medium and the color changed (first column). Negative, the tested isolate only grows in the absence of colistin (second column). Positive, the tested isolate grows in the presence and absence of colistin (third column)

Rapid ResaPolymyxin Acinetobacter/Pseudomonas NP test

The experimental procedure was performed according to the previously described protocol [15]. Briefly, the colistin-containing Mueller Hinton broth (MHB, OXOID, UK) solution was prepared with an initial concentration of $4.16 \mathrm{mg} / \mathrm{L}$. Then, a $180 \mu \mathrm{l}$ colistin-free MHB solution and colistin-containing MHB solution were added to lines A and B of a 96-well polystyrene micro test plate, respectively. For each isolate, $20 \mu \mathrm{l}$ of the bacterial suspension at a 3.5 McFarland optical density $\left(\sim 1 \times 10^{9} \mathrm{CFU} / \mathrm{mL}\right)$ was inoculated in parallel into two wells, with and without colistin. The bacterial suspension was mixed with the medium by pipetting up and down. The final concentration of colistin was $3.75 \mathrm{mg} / \mathrm{L}$. In the same way, $20 \mu \mathrm{l}$ of $0.85 \% \mathrm{NaCl}$ was used as an aseptic control, $20 \mu \mathrm{l}$ of the colistin-susceptible isolate (E. coli ATCC 25922 and P. aeruginosa ATCC 27853) suspension was used as negative control; and $20 \mu$ of the colistin-resistant isolate (the clinical isolates of Morgan, inherent resistance to polymyxin) suspension was used as a positive control. After testing several isolates, we ensured that the color-transfer of colistin suspension and the mixing of bacterial suspension in the micro test plate were completed within $15 \mathrm{~min}$. The inoculated tray was incubated at $35 \pm 2{ }^{\circ} \mathrm{C}$ for $3 \mathrm{~h}$. Then, $22 \mu \mathrm{l}$ of the resazurin reagent PrestoBlue ${ }^{\oplus}$ (ThermoFisher Scientific, US, final concentration is $10 \% \mathrm{~V} / \mathrm{V}$ ) was added per well and each well was mixed by pipetting up and down. Finally, the tray was visually inspected every 15 min within $1 \mathrm{~h}$. Susceptibility of colistin is determined by the color changes, where discoloration indicates that the strain is colistin-resistant, while the lack of discoloration indicates that the strain is colistin-susceptible [15]. All experiments were performed in triplicate.

The test was considered to be positive (i.e., purple or pink) if the colistin-resistant isolate was viable in presence of colistin, or negative (i.e., blue) if the colistinsusceptible isolate was not viable in presence of colistin. The Rapid ResaPolymyxin Acinetobacter/Pseudomonas NP test interpretation is illustrated in Fig. 1.

\section{Abbreviations}

ATCC: American Type Cultures Collection; BMD: Broth Microdilution; CFU: Colony Forming Unit; CLSI: Clinical and Laboratory Standards Institute; EUCAST: European Committee of Antibiotic Susceptibility Testing; MDR: Multidrug resistance; ME: Major Error; MHB: Mueller Hinton broth; MIC: Minimal Inhibitory Concentration; VME: Very Major Error

\section{Acknowledgements}

Not applicable.

\section{Authors' contributions}

$\mathrm{HJ}$ conducted the experiments, analyzed the data and wrote the manuscript. RF participated in experiments and writing. JL and XT provided colistinresistant strains and participated in analysis of results. $Y Z$ participated in analysis of results. LC helped design the study. JC and TZ designed the study and corrected the manuscript. All authors read and approved the manuscript.

\section{Funding}

This study was supported by the National Natural Science Foundations of China (No. 81971986) and the General Scientific Research Project of the Education Department of Zhejiang Province of China (No. Y201942210) and the Planned Science and Technology Project of Wenzhou (no. Y20170204).

\section{Availability of data and materials}

All data generated or analyzed during this study are included in this published article. 
Ethics approval and consent to participate

Not applicable.

\section{Consent for publication}

Not applicable.

\section{Competing interests}

The authors declare that they have no competing interests.

Received: 6 September 2019 Accepted: 24 December 2019

Published online: 08 January 2020

\section{References}

1. Gregoire N, Aranzana-Climent V, Magreault S, Marchand S, Couet W. Clinical pharmacokinetics and pharmacodynamics of Colistin. Clin Pharmacokinet. 2017:56(12):1441-60.

2. Brooks LE, UI-Hasan S, Chan BK, Sistrom MJ. Quantifying the Evolutionary Conservation of Genes Encoding Multidrug Efflux Pumps in the ESKAPE Pathogens To Identify Antimicrobial Drug Targets. mSystems. 2018;3(3): e00024-18.

3. Li J, Nation RL, Turnidge JD, Milne RW, Coulthard K, Rayner CR, Paterson DL. Colistin: the re-emerging antibiotic for multidrug-resistant gram-negative bacterial infections. Lancet Infect Dis. 2006:6(9):589-601.

4. Olaitan AO, Diene SM, Kempf M, Berrazeg M, Bakour S, Gupta SK, Thongmalayvong B, Akkhavong K, Somphavong S, Paboriboune P, et al. Worldwide emergence of colistin resistance in Klebsiella pneumoniae from healthy humans and patients in Lao PDR, Thailand, Israel, Nigeria and France owing to inactivation of the PhoP/PhoQ regulator mgrB: an epidemiological and molecular study. Int J Antimicrob Agents. 2014;44(6): 500-7.

5. Wang X, Liu Y, Qi X, Wang R, Jin L, Zhao M, Zhang Y, Wang Q, Chen H, Wang $\mathrm{H}$. Molecular epidemiology of colistin-resistant Enterobacteriaceae in inpatient and avian isolates from China: high prevalence of mcr-negative Klebsiella pneumoniae. Int J Antimicrob Agents. 2017;50(4):536-41.

6. CLSI. Performance Standards for Antimicrobial Susceptibility Testing. 29th ed. CLSI supplement M100. Wayne: Clinical and Laboratory Standards Institute; 2019.

7. The European Committee on Antimicrobial Susceptibility Testing. Breakpoint tables for interpretation of MICs and zone diameters. Version 9.0; 2019. http://www.eucast.org.

8. Humphries RM. Susceptibility testing of the Polymyxins: where are we now? Pharmacother J Human Pharmacol Drug Ther. 2015;35(1):22-7.

9. Vasoo S, Munson E. Susceptibility testing for the Polymyxins: two steps back, three steps forward? J Clin Microbiol. 2017;55(9):2573-82.

10. Humphries RM, Hindler JA, Epson E, Horwich-Scholefield S, Miller LG Mendez J, Martinez JB, Sinkowitz J, Sinkowtiz D, Hershey C, et al. Carbapenem-resistant Enterobacteriaceae detection practices in California: what are we missing? Clin Infect Dis. 2018;66(7):1061-7.

11. Satlin MJ. The Search for a Practical Method for Colistin Susceptibility Testing: Have We Found It by Going Back to the Future? I Clin Microbiol. 2019;57(2):e01608-18.

12. Matuschek E, Ahman J, Webster C, Kahlmeter G. Antimicrobial susceptibility testing of colistin - evaluation of seven commercial MIC products against standard broth microdilution for Escherichia coli, Klebsiella pneumoniae, Pseudomonas aeruginosa, and Acinetobacter spp. Clin Microbiol Infect. 2018:24(8):865-70.

13. Ezadi F, Ardebili A, Mirnejad R. Antimicrobial Susceptibility Testing for Polymyxins: Challenges, Issues, and Recommendations. J Clin Microbiol. 2019;57(4):e01390-18.

14. Nordmann P, Jayol A, Poirel L. Rapid detection of Polymyxin resistance in Enterobacteriaceae. Emerg Infect Dis. 2016;22(6):1038-43.

15. Lescat M, Poirel L, Tinguely C, Nordmann P. A Resazurin Reduction-Based Assay for Rapid Detection of Polymyxin Resistance in Acinetobacter baumannii and Pseudomonas aeruginosa. J Clin Microbiol. 2019;57(3): e01563-18.

16. Jayol A, Nordmann P, Lehours P, Poirel L, Dubois V. Comparison of methods for detection of plasmid-mediated and chromosomally encoded colistin resistance in Enterobacteriaceae. Clin Microbiol Infect. 2018;24(2):175-9.

17. Lu H, Wang C, Dong G, Xu C, Zhang X, Liu H, Zhang M, Cao J, Zhou T. Prevalence and Molecular Characterization of Escherichia coli Clinical Isolates
Carrying mcr-1 in a Chinese Teaching Hospital from 2002 to 2016 Antimicrob Agents Chemother. 2018;62(9):e02623-17.

18. Lin J, Xu C, Fang R, Cao J, Zhang X, Zhao Y, Dong G, Sun Y, Zhou T. Resistance and hetero-resistance to colistin in Pseudomonas aeruginosa isolates from Wenzhou, China. Antimicrob Agents Chemother. 2019;63(10): e00556-19.

19. Lutgring JD, Kim A, Campbell D, Karlsson M, Brown AC, Burd EM. Evaluation of the MicroScan Colistin Well and Gradient Diffusion Strips for Colistin Susceptibility Testing in Enterobacteriaceae. J Clin Microbiol. 2019;57(5): e01866-18.

20. Chew KL, La M-V, Lin RTP, Teo JWP, Munson E. Colistin and Polymyxin B susceptibility testing for Carbapenem-resistant and mcr-positive Enterobacteriaceae: comparison of Sensititre, MicroScan, Vitek 2, and Etest with broth microdilution. J Clin Microbiol. 2017;55(9):2609-16.

21. Pillonetto M, Mazzetti A, Becker GN, Siebra CA, Arend L, Barth AL. Low level of polymyxin resistance among nonclonal mcr-1-positive Escherichia coli from human sources in Brazil. Diagn Microbiol Infect Dis. 2019;93(2):140-2.

\section{Publisher's Note}

Springer Nature remains neutral with regard to jurisdictional claims in published maps and institutional affiliations.

Ready to submit your research? Choose BMC and benefit from:

- fast, convenient online submission

- thorough peer review by experienced researchers in your field

- rapid publication on acceptance

- support for research data, including large and complex data types

- gold Open Access which fosters wider collaboration and increased citations

- maximum visibility for your research: over $100 \mathrm{M}$ website views per year

At $\mathrm{BMC}$, research is always in progress.

Learn more biomedcentral.com/submission 